\title{
Comparison of the effects of perinatal and neonatal administration of sodium ferulate on repair following excitotoxic neuronal damages induced by maternal oral administration of monosodium glutamate at a late stage of pregnancy
}

\author{
Yongping Zhang ${ }^{1}$, Lijian Yu ${ }^{1}$, Rundi Ma ${ }^{1}$, Xiaoyu Zhang ${ }^{1,2}$, Tingxi Yu ${ }^{1,3}$ \\ ${ }^{1}$ Key Laboratory of Mrine Materia Medica, Guangdong Ocean University, Zhanjiang, China \\ ${ }^{2}$ Department of Otorhinolaryngology_-Head and Neck Surgery, School of Medicine, University of Maryland, Baltimore, USA \\ ${ }^{3}$ Shanghai Pediatric Medical Research Institute, Xinhua Hospital, Shanghai Jiao Tong University, Shanghai, China \\ Email: yutingxi@yahoo.com,ywyj9578@sohu.com
}

Received 23 April 2012; revised 23 May 2012; accepted 1 June 2012

\begin{abstract}
Objective: Our previous studies have revealed that ferulic acid (FA) and sodium ferulate (SF) show significant protective effect on excitotoxicity. The present study was conducted to compare its potential favorable effects of maternal, newborn, and both maternal and newborn intraperitoneal (ip) injection of SF on repair following excitotoxic neuronal damages induced by monosodium glutamate (MSG). Methods: The maternal mice were assigned randomly into seven groups ( $n=10$ animals in each group): control, 3SF, 20SF, 23SF, MSG, MSG + 3SF, MSG + 20SF, MSG + 23SF groups. The mice at 17 days of pregnancy were treated with or without MSG ( $2.0 \mathrm{~g} / \mathrm{kg}$ body weight, ig, once) or/and SF $(40 \mathrm{mg} / \mathrm{kg}$ body weight, ip), and their offerings treated with or without SF. And then their filial behaviors and hippocampal histopathology were studied. Results: The results showed that maternal, newborn, and both maternal and newborn administration of SF facilitated their filial brain repair, and attenuated the behavioral disorders and histopathological damages of their filial mice in MSG + 3SF, MSG + 20SF, and MSG + 23SF groups in varying degrees. However, the best effects were detected in the filial mice in MSG + 23SF group. Conclusion: Both maternal and newborn administration of SF is conducive to the filial neuronal repair following excitotoxic damages induced by glutamate.
\end{abstract}

Keywords: Perinatal-Neonatal; Excitotoxicity; Sodium Ferulate; Neuronal Repair; Mice

\section{INTRODUCTION}

Excitatory amino acids (EAAs) are utilized by nearly every information - bearing circuit in the brain, and during development. EAAs play a pivotal role in learning, memory, and brain plasticity. Exitotoxicity is an important and well accepted theory [1-3] proposed by Olney in 1971 [4] to explain the pathophysiology of brain ischemia. Exitotoxicity, defined as excessive exposure to the neurotransmitter glutamate or overstimulation of its membrane receptors, has been implicated as one of the key factors constributing to neuronal injury and death in a wide range of both acute and chronic neurodegenerative disorders. Neurodegenerative disorders, including Alzheimer's disease, Parkingson's disease, Huntington's chorea, HIV-associated dementia, multiple sclerosis, amyotrophic lateral sclerosis, and glaucoma, are caused by different mechanisms but may share a final common pathway to neuronal injury due to overstimulation of glutamate receptors, especially of the N-methyl-D-aspartate (NMDA) subtype [5]. Therefore, antagonizing exitotoxicity is a particularly attractive target for neuroprotective or brain repair efforts. In context, we have successfully established an experimental model of excito-toxic brain injury following exposure to glutamate, in which maternal ig excessive administration of MSG at a late stage of pregnancy results in a series of behavioral disorders, and obvious histopathological brain lesion in their offspring $[6,7]$.

Ferulic acid (FA), 3-(4-hydroxy-3-methoxyphenyl)-2propenoic acid (Figure 1(a)), was isolated from several traditional Chinese medicines and herbs, such as Felula assafoetida L., Ligusticum chuanxiong Hort., Equisetum hiemale L., Allium cepa L., Raphanus sativus L., rice bran, and other plants [8]. FA is water-insoluble, but its sodium salt, sodium ferulate (SF) (Figure 1(b)), is watersoluble, stable, and can be prepared by chemical synthesis 


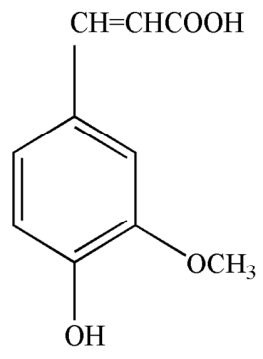

(a)

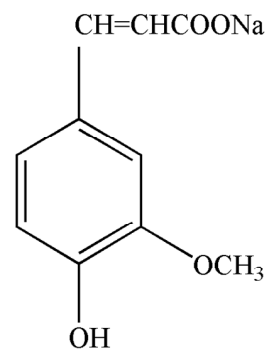

(b)
Figure 1. Chemical structure of ferulic acid (a) and sodium ferulate (b).

[9]. FA shows a lot of biological activities, including antioxidant $[10,11]$, anti-inflammatory $[12,13]$, and hypotensive effect $[14,15]$. Chinese scientists have contributed a lot to its investigation, and as an available bloodactivating and stasis-eliminating component, it has been extensively applied to the treatment of vascular diseases of heart and brain, and obtained excellent efficiency in China [16].

Long-term administration of FA induces resistance to beta-amyloid peptide toxicity in the brain, suggesting that FA may be a useful chemopreventive agent against Alzheimer's disease [17]. FA has sedative effect [18], and the plants containing FA were employed, for instance, in the treatment of headache and irritability, apoplexy, stasis, and etc. $[19,20]$. Our previous work demonstrates that the maternal ig excessive administration of MSG at a late stage of pregnancy results in a series of behavioral disturbance, and obvious histopathological lesion in hippocampus in the filial mice [6], and the simultaneous administration of SF reverses the effects of MSG on them [7]. Our recent work provides evidences that SF shows the neuroprotective and neurogenesisenhancing actions in vitro and in vivo [21]. The present study was conducted to compare its potential favorable effects of maternal, newborn, and both maternal and newborn ip injection of SF on brain repair following excitotoxic neuronal damages induced by maternal oral administration of MSG at a late stage of pregnancy.

\section{MATERIALS AND METHODS}

\subsection{Chemical and Drug}

MSG was purchased from Shanghai Bio Life Science \& Technology Co., Ltd. (China); SF was purchased from Yaoyou Pharmaceutic Co., Ltd (Chongqing, China).

\subsection{Instruments}

The apparatus of open field test (type ZIL-2) was purchased from Institute of Materia Medica, Chinese Academy of Medical Sciences (Beijing, China), and MG-3- type Y-maze stimulator purchased from Biomedical Instrument Factory of Zhangjiagang (Jiangsu Province, China).

\subsection{Animal}

Kunming (KM) mice, 8 weeks old, purchased from the Experimental Animal Center of Guangdong Medical College (Zhanjiang, China), were used in this study. The mice were provided with standard mouse food and chlorinated water ad libitum and kept on a reversed 12:12 h light-dark cycle at room temperature of $22^{\circ} \mathrm{C} \pm 1{ }^{\circ} \mathrm{C}$. All the animals were treated in compliance with "Guidance Suggestion for the Care and Use of Laboratory Animals" issued by The Ministry of Science and Technology of China in 2006.

\subsection{Protocols of Animal Tests}

Animals were housed one male and two female mice to each cage. The pregnant females were separated. The time of appearance of vaginal plug was recorded as day 1 of pregnancy. The maternal mice at 17 days of pregnancy were assigned randomly into seven matched groups $(n=$ 10 animals in each group): control, 3SF, 20SF, 23SF, $\mathrm{MSG}, \mathrm{MSG}+3 \mathrm{SF}, \mathrm{MSG}+20 \mathrm{SF}, \mathrm{MSG}+23 \mathrm{SF}$ groups. The mice in MSG group were given MSG $(2.0 \mathrm{~g} / \mathrm{kg}$ body weight, once) intragastrically (ig) at 17 days of pregnancy. The mice in MSG + 3SF and 3SF groups were given MSG (2.0 g/ $/ \mathrm{kg}$ body weight, ig, once ) or physiologic saline at 17 days of pregnancy, respectively, and $4 \mathrm{~h}$ later given SF ( $40 \mathrm{mg} / \mathrm{kg}$ body weight, ip, once daily, for three days) at $17-19$ days of pregnancy. The mice in MSG +20 SF and 20SF groups were given MSG (2.0 $\mathrm{g} / \mathrm{kg}$ body weight, ig, once ) or physiologic saline at 17 days of pregnancy, respectively, and the newborn offspring were given SF $(40 \mathrm{mg} / \mathrm{kg}$ body weight, ip, once daily, for 20 days ) from the first day of birth. The mice in $\mathrm{MSG}+23 \mathrm{SF}$ and 23SF groups were given MSG (2.0 $\mathrm{g} / \mathrm{kg}$ body weight, ig, once) or physiologic saline at 17 days of pregnancy, respectively, and $4 \mathrm{~h}$ later given SF (40 mg/kg body weight, ip, once daily, for three days) at 17 - 19 days of pregnancy, and then the newborn offspring were given SF $(40 \mathrm{mg} / \mathrm{kg}$ body weight, ip, once daily, for 20 days ) from the first day of birth. The mice in control were given physiologic saline ig or ip instead of MSG or SF. Their filial behaviors and hippocampal histopathology were studied.

\subsection{Open Field Test}

The open field test measures the animal's reaction to a novel environment [22]. The test was performed at 31 days of age. Total locomotor activity in $3 \mathrm{~min}$ was calculated. 


\subsection{Test of Y-Maze Discrimination Learning}

Test of Y-maze discrimination learning was carried out by Xu's method [23]. Test of Y-maze discrimination learning for 6 consecutive days was performed on day 32 and 52 after the birth. The results were recorded as correct responses (means $\pm \mathrm{SEM}$ ) of 20 responses.

\subsection{Rope Crawling Test}

The rope crawling test measures an animal's cooperation movement ability under high altitude stress [6]. The 31day-old mice were placed on a $2 \mathrm{~m}$ long rope, $1.5 \mathrm{~m}$ above the ground, and then the behaviors of the mice were observed for $3 \mathrm{~min}$. The mice that could grasp and crawl along the rope were deemed to have "succeeded" in the test, and those mice that could either not grasp a rope tightly or grasped the rope tightly but could not crawl along "failed" in the test.

\subsection{Examination of Histopathology}

The histopathology changes of the animal brains were examined for three times: on day 1 after the birth, at 21 days of age, and 53 days of age. The unselected 4 filial mice of each group were anaesthetized ip with sodium pentobarbital $(60 \mathrm{mg} / \mathrm{kg}$ ), and sacrificed by perfusion fixation of the central nervous system (CNS) with 10\% formalin. The whole brains were excised carefully, and further fixed in 10\% formalin for a week. The hippocampal region of each animal was sectioned at $4 \mathrm{~mm}$ and stained with hematoxylin and eosin. The sections across hippocampus region were examined by light microscopy.

\subsection{Statistical Analyses}

All data were statistically processed using SPSS 11.0 software (SPSS Inc., Chicago, IL, USA) and expressed as means \pm SEM. The repeated-measures analysis with a compound symmetry variance-covariance structure model was used to analyze the data from test of Y-maze discrimination learning, and Student's $t$-test used to analyze the data from open field test. The $\chi^{2}$ square test was used to analyze the data from the rope crawling test. A P value less than 0.05 was considered statistically significant.

\section{RESULTS}

\subsection{The Different Effects of Maternal, Newborn, and Both Maternal and Newborn Administration of SF on MSG-Induced Hyperactivity in the Filial Mice}

As shown in Figure 2, the locomotor activity of the filial mice in $3 \mathrm{SF}, 20 \mathrm{SF}, 23 \mathrm{SF}$ groups was similar to that in the control $(\mathrm{P}>0.05)$, and a MSG-induced hyperactivity observed in the filial mice from mothers treated with
MSG $(\mathrm{P}<0.01)$. In contrast, the locomotor activity of the filial mice from mothers in MSG $+3 \mathrm{SF}$ and MSG + 23SF groups was less than that of the filial mice from mothers treated with MSG. These findings suggest that SF had no obvious effect on locomotion and exploration, rather than, countered the effect of MSG on those of the filial mice from mothers treated with MSG. In this regard, the repair effects of maternal, and both maternal and newborn administration of SF on MSG-induced hyperactivity is more effective than the effect of newborn administration of SF.

\subsection{The Different Effects of Maternal, Newborn, and Both Maternal and Newborn Administration of SF on Animal's Cooperation of Movement Ability under High Altitude Stress in the Filial Mice}

As shown in Table 1, the cooperative movement ability under high-altitude stress of 31-day-old filial mice in 3SF, 20SF, 23SF groups was similar to that in the control $(\mathrm{P}>0.05)$, and the cooperative movement ability under high-altitude stress in 31-day-old filial mice from mothers treated with MSG was significantly less than that of the control $(\mathrm{P}<0.01)$. In contrast, cooperative movement ability under high-altitude stress of 31-day-old filial mice from mothers in MSG + 23SF group was significantly improved and close to control. The results suggest that administration of SF had no obvious effects on the cooperation of movement ability in filial mice under highaltitude stress, apart from countering the effects of MSG. In this regard, the repair effect of both maternal and newborn administration of SF on MSG-induced disturbance of cooperative movement ability under high-altitude stress is the most effective than those of maternal and newborn administration of SF.

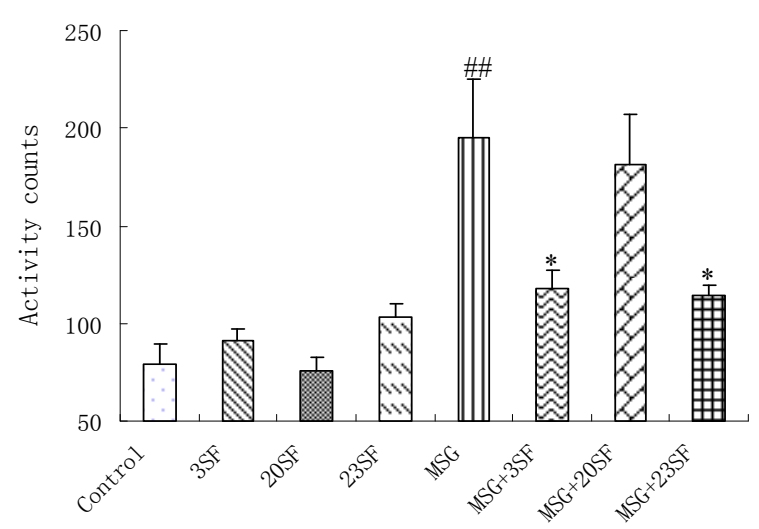

The open field test measures the animal's reaction to a novel environment. The test was performed at 31 days of age. Total locomotor activity in 3 min was calculated. ${ }^{\# \#} \mathrm{P}<0.01$ vs. control; ${ }^{*} \mathrm{P}<0.05$ vs. MSG.

Figure 2. The different effects of maternal, neonatal, and both maternal and neonatal administration of SF on MSG-induced hyperactivity in the filial mice. 
Table 1. Different effects of maternal, newborn, and both maternal and newborn administration of SF on MSG-induced disturbance of cooperation of movement ability under high-altitude stress in the filial mice.

\begin{tabular}{ccc}
\hline Groups & Number of mice & $\begin{array}{c}\text { Number of mice crawling } \\
\text { along a rope }\end{array}$ \\
\hline Control & 12 & 11 \\
MSG & 12 & $5^{\# \#}$ \\
3 SF & 13 & 13 \\
20 SF & 12 & 11 \\
23 SF & 13 & 11 \\
MSG +3 SF & 12 & 8 \\
MSG +20 SF & 13 & 7 \\
MSG $+23 S F$ & 13 & $10^{*}$ \\
\hline
\end{tabular}

$\# \mathrm{P}<0.01$ vs. control; ${ }^{*} \mathrm{P}<0.05$ vs. MSG.

\subsection{The Different Effects of Maternal, Newborn, and Both Maternal and Newborn Administration of SF on the Capability of Y-Maze Discrimination Learning in the Filial Mice}

As shown in Figure 3, the capability of Y-maze discrimination learning of 32-, 52-day-old filial mice in 3SF, 20SF, 23SF groups was similar to that in the control (P > 0.05 ), and the capability of Y-maze discrimination learning of 32- and 52-day-old filial mice from mothers treated with MSG was significantly less than that of the control $(\mathrm{P}<0.01)$. In contrast, the capability of Y-maze discrimination learning of 32- and 52-day-old filial mice in $\mathrm{MSG}+3 \mathrm{SF}$ and $\mathrm{MSG}+23 \mathrm{SF}$ groups was significantly improved and close to control. In this regard, the repair effect of both maternal and newborn administration of $\mathrm{SF}$ on the capability of Y-maze discrimination learning is the most effective among the maternal, newborn, and both maternal and newborn administration.

\subsection{The Different Effects of Maternal, Newborn, and Both Maternal and Newborn Administration of SF on the Histopathology of the Animal Brains in the Filial Mice}

Hippocampal histomorphological changes in filial mice were observed using hematoxylin and eosin staining. As shown in Figures 4-6, MSG-induced lesions were characterized by intracellular edema, neuronal degeneration and necrosis in the hippocampi of the neonatal filial mice, and characterized by neuronal degeneration and necrosis, and hyperplasia in the hippocampi of the 21- and 53day-old filial mice. A slight MSG-induced hippocampal histomorphological lesions was detected in the hippocampi of the neonatal filial mice from mothers in MSG + $3 \mathrm{SF}$ group, and of the 21- and 53-day-old filial mice in

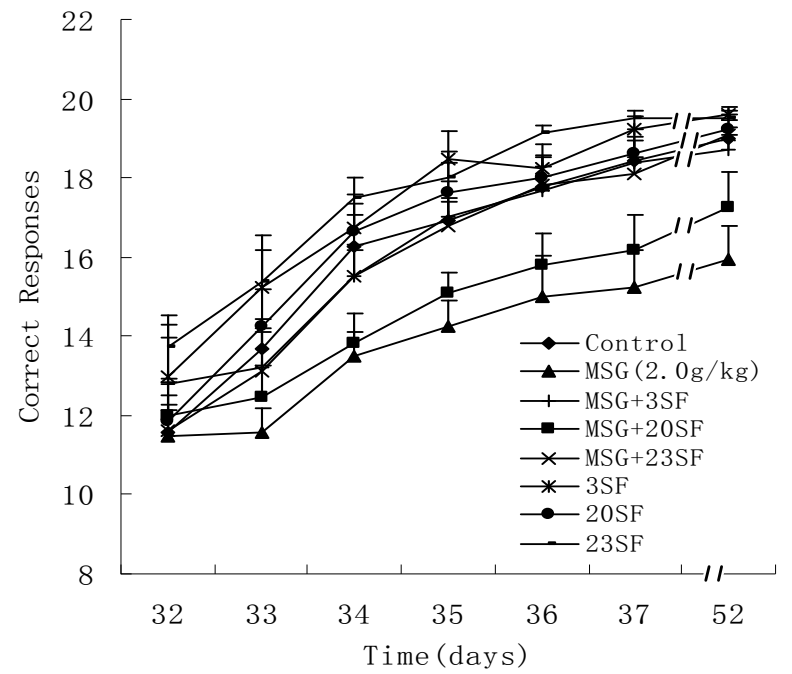

Test of Y-maze discrimination learning for 6 consecutive days was performed on days 32 and 52 after the birth. The results were recorded as correct responses (means \pm SEM) of 20 responses. MSG vs. control, $\mathrm{P}$ $<0.01$; $3 \mathrm{SF}$ vs. control, $\mathrm{P}>0.05 ; 20 \mathrm{SF}$ vs. control, $\mathrm{P}>0.05 ; 23 \mathrm{SF}$ vs. control, $\mathrm{P}>0.05 ; \mathrm{MSG}+3 \mathrm{SF}$ vs. MSG, $\mathrm{P}<0.05 ; \mathrm{MSG}+20 \mathrm{SF}$ vs. MSG, $\mathrm{P}>0.05 ; \mathrm{MSG}+23 \mathrm{SF}$ vs. MSG, $\mathrm{P}<0.01$.

Figure 3. The different effects of maternal, neonatal, and both maternal and neonatal administration of SF on the capability of Y-maze discrimination learning in the filial mice.

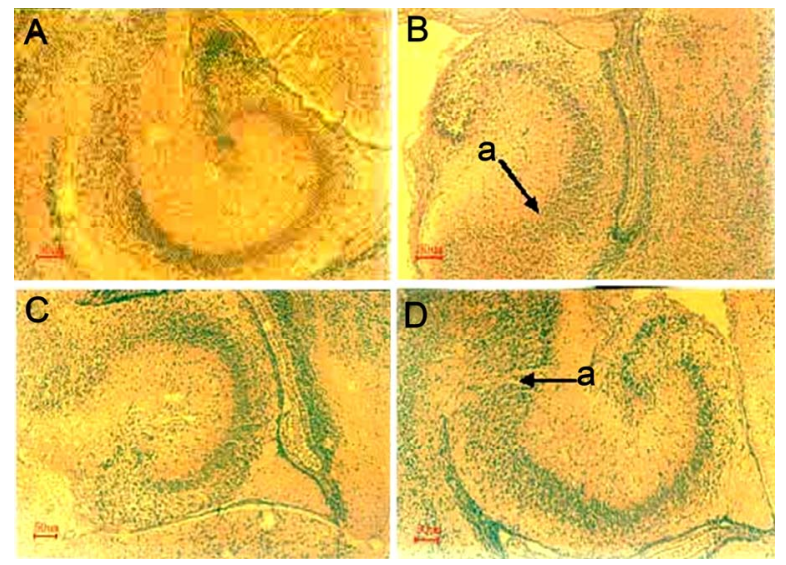

The maternal mice were treated with or without MSG or/and SF as described in "Materials and Methods". The histopathology changes of the neonatal brains were examined on day 1 after the birth. The hippocampal region of each animal was sectioned at $4 \mu \mathrm{m}$ and stained with hematoxylin and eosin. The sections across hippocampus region were examined by light microscopy. Scale bar, $50 \mu \mathrm{m}$. A. Control; B. MSG; C. 3SF; D. $\mathrm{MSG}+3 \mathrm{SF}$. a. Neuronal degeneration and necrosis.

Figure 4. MSG-induced hippocampal histomorphological lesions in the neonatal mice and the repair effect of administration of SF.

MSG +20 SF group. However, no significant histopathological damages was detected in the hippocampi of the neonatal, 21- and 53-day-old filial mice in 3SF, 20SF, 23SF groups, and of the 21- and 53-day-old filial mice in $\mathrm{MSG}+3 \mathrm{SF}$ and MSG $+23 \mathrm{SF}$ groups. Results from this study also reflected the dynamics of brain repair following MSG-induced excitotoxic histopathologycal damages. 
In this regard, the repair effects of maternal, and both maternal and newborn administration of SF on the MSGinduced hippocampal histomorphological lesions in the filial mice are more effective than the effect newborn administration.

\section{DISCUSSION}

The development of mice is an example of experimental mammalian embryology, and mouse development is the basis for the study of human ontogeny. In the present study, the purpose of the method given orally MSG was to better mimic the entry approach of MSG food additive into the human food chain; and MSG was given at a late stage of pregnancy when mouse embryonic CNS had basically taken shape, thus the tests could mimic the effects of MSG poisoning on children's mental development and behavior, and examine the effects of SF on repair of neuronal damages induced by glutamate.

Behavioral experiments showed that capabilities of animal's cooperation of movement under high altitude stress and of Y-maze discrimination learning were significantly reduced, while the locomotor activity was significantly enhanced in the filial mice from mothers treated with MSG; in contrast, the cooperative movement ability under high-altitude stress of 31-day-old filial mice from mothers in MSG + 23SF group and the capability of Y-maze discrimination learning of 32- and 52-day-old filial mice in $\mathrm{MSG}+3 \mathrm{SF}$ and $\mathrm{MSG}+23 \mathrm{SF}$ groups were significantly improved $(\mathrm{P}<0.05, \mathrm{P}<0.01$ vs. $\mathrm{MSG}$,
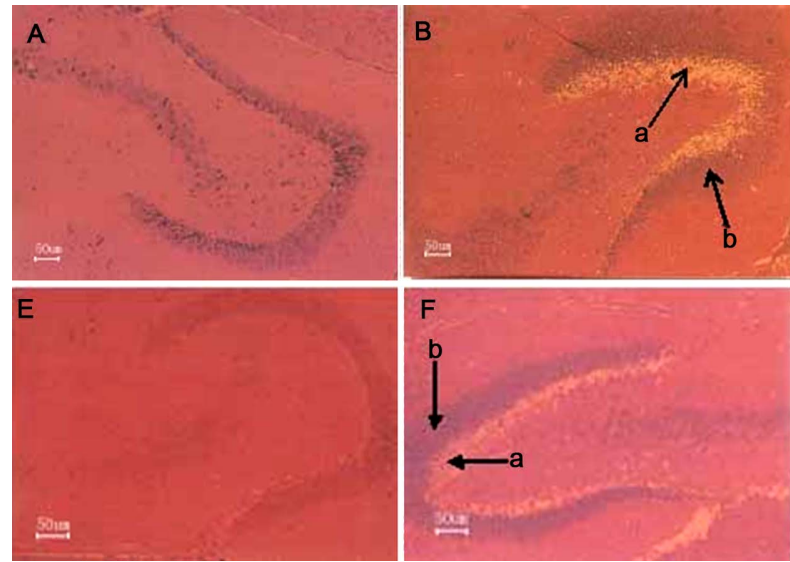
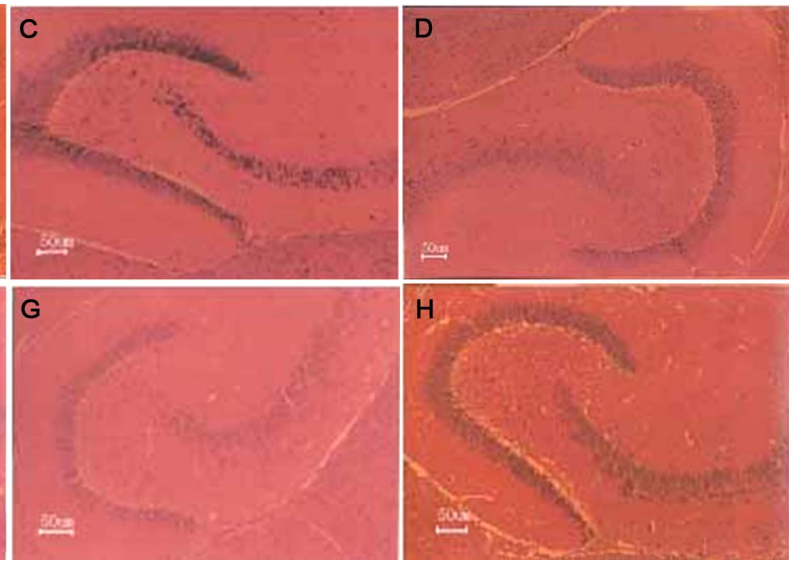

The maternal mice were treated with or without MSG or/and SF, and their offspring treated with SF as described in "Materials and Methods". The histopathology changes of the brains of the 21-day-old filial mice were examined. The hippocampal region of each animal was sectioned at $4 \mu \mathrm{m}$ and stained with hematoxylin and eosin. The sections across hippocampus region were examined by light microscopy. Scale bar, $50 \mu \mathrm{m}$. A. Control; B. MSG; C. 3SF; D. MSG + 3SF; E. 20SF; F. MSG + 20SF; G. 23SF; H. MSG + 23SF. a. Neuronal degeneration and necrosis; b. Hyperplasia.

Figure 5. Repair effect of administration of SF on MSG-induced hippocampal histomorphological lesions in the 21-day-old filial mice.
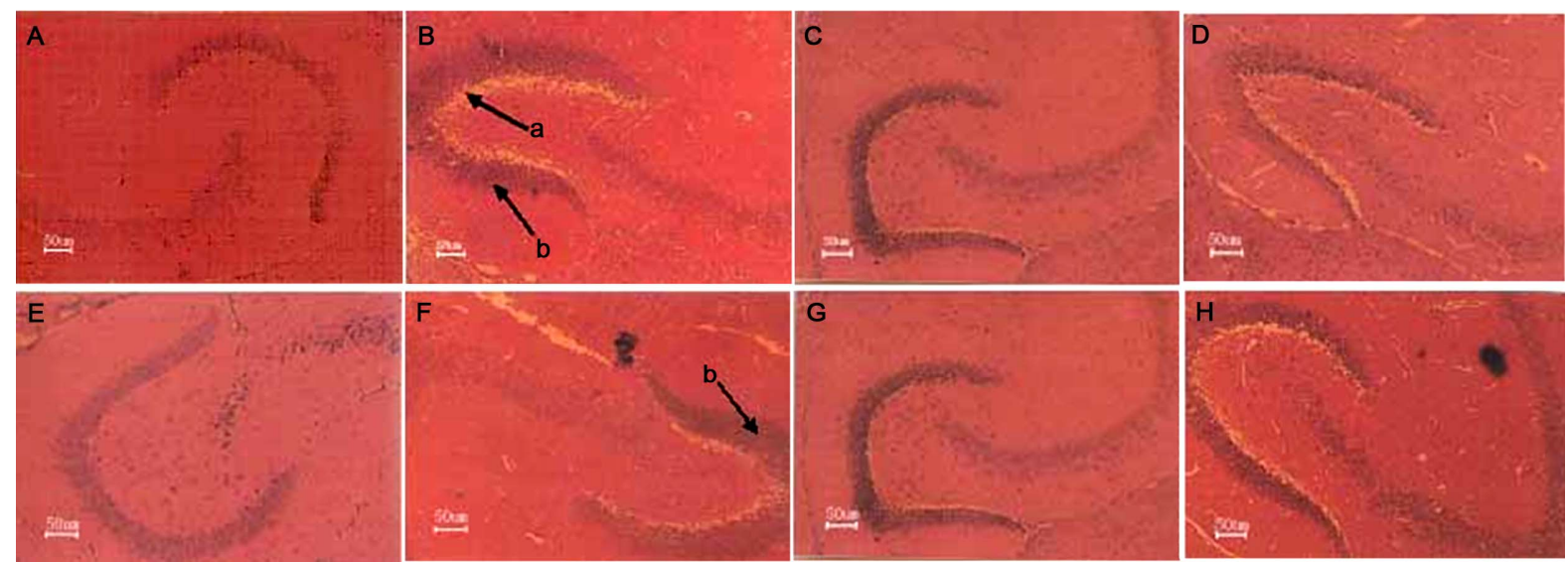

The maternal mice were treated with or without MSG or/and SF, and their offspring treated with SF as described in "Materials and Methods". The histopathology changes of the brains of the 53-day-old filial mice were examined. The hippocampal region of each animal was sectioned at $4 \mu \mathrm{m}$ and stained with hematoxylin and eosin. The sections across hippocampus region were examined by light microscopy, Scale bar, $50 \mu \mathrm{m}$. A. Control; B. MSG; C. 3SF; D. MSG + 3SF; E. 20SF; F. MSG + 20SF; G. 23SF; H. MSG + 23SF. a. Neuronal degeneration and necrosis; b. Hyperplasia.

Figure 6. Repair effect of maternal administration of SF on MSG-induced hippocampal histomorphological lesions in the 53-day-old filial mice. 
respectively) and close to controls, while the locomotor activity of the filial mice from mothers in MSG + 3SF and MSG $+23 \mathrm{SF}$ groups was significantly reduced. Obviously both maternal and newborn administration of SF is the most conducive to the filial behavioral functional recovery from excitotoxic neuronal damages induced by glutamate.

Histopathological examination showed that MSG-induced lesions were characterized by intracellular edema, neuronal degeneration and necrosis, and hyperplasia in the hippocampi of the filial mice from mothers treated with MSG. A slight MSG-induced hippocampal histomorphological lesions was also detected in the hippocampi of the neonatal filial mice in MSG + 3SF group, and of the 21- and 53-day-old filial mice in MSG + 20SF group. However, no significant histopathological damages was detected in the hippocampi of the neonatal, 21and 53-day-old filial mice in 3SF, 20SF, 23SF groups, and of the 21- and 53-day-old filial mice in MSG + 3SF and MSG $+23 \mathrm{SF}$ groups. Therefore, the repair effects of maternal, and both maternal and newborn administration of SF on the MSG-induced hippocampal histomorphological lesions are more effective than the effect of newborn administration.

It is well recognized that excessive activation of EAA receptors can initiate a series of intracellular biochemical events that cause neuronal damages referred to as excitotoxicity, and excitotoxic neuronal injury in the immature brain can be blocked by selective antagonists of EAA receptors [24-28].

It is possible that an adult female during pregnancy might have either a disturbance in EAA metabolism or exogenous excess uptake of EAAs resulting in markedly elevated EAA concentrations in the blood and, therefore might expose the fetus in utero to high enough blood levels of EAAs to cause subtle excitotoxic brain damages by interaction of EAAs with NMDA receptors during a period of NMDA receptor hypersensitivity. It is also possible that both the mother and fetus might have the metabolic abnormality of EAAs so that the fetus, being compromised in dealing with the excessive EAAs received from the maternal blood, would develop exceedingly high blood and brain levels of the toxin [29].

Our previous work reveals that maternal oral administration of $\left[{ }^{3} \mathrm{H}\right]$ glutamate at a late stage of pregnancy results in uptake of radioactivity to mouse fetal brain. The maternal ig excessive administration of MSG at a late stage of pregnancy results in a series of behavioral disorders, including MSG-induced hyperactivity that is reminiscent of what is seen in the children suffered from attention deficit hyperactivity disorder (ADHD), and obvious histopathological lesions in hippocampus in the filial mice. Simultaneous administration of SF obviously inhibits the uptake of labeled glutamate in fetal brain, and counters the effects of MSG on behavior, histopathology, genetic toxicity, and expression of apoptosis-related gene. The results suggest that SF is a novel competitive NMDA receptor antagonist and neuroprotector. Therefore, maternal administration of SF has potent protective effects against glutamate-induced neurotoxicity in their filial mice $[6,7]$. Our present work demonstrates that maternal, and both maternal and newborn subsequent administration of SF facilitates the repair following excitotoxic neuronal damages induced by maternal oral administration of MSG at a late stage of pregnancy.

Our recent experimental results obtained from in vitro tests show that SF treatment promotes the proliferation of PC12 cells in the concentration range of $5-320 \mathrm{mM}$, and induces PC12 cells to differentiate to more mature cells with the morphological characteristics and molecular marker of neuronal-like cells; the results obtained from in vivo tests show that SF treatment may up-regulate the expressions of nerve growth factor and brainderived neurotrophic factor, and induce the proliferation of neural stem cell/neural progenitor cells in the hippocampi of chronic mild stress-induced depression-like model rats [21]. Taken together, promotive effect of brain repair of SF is related to neuroprotective and neurogenesisenhancing effects of SF, but may mainly result from its neurogenesis-enhancing effect.

\section{ACKNOWLEDGEMENTS}

The authors would like to thank Dr. Depu Yu for his great encouragement and continuous promotion.

\section{REFERENCES}

[1] Choi, D.W. (1990) Methods for antagonizing glutamate neurotoxicity. Cerebrovascular \& Brain Metabolism Reviews, 2, 105-147.

[2] Greenamyre, J.T. and Porter, R.H.P. (1994) Anatomy and physiology of glutamate in the CNS. Neurology, 44, S7S13.

[3] Jay, T.M. (1996) Neurotransmitter systems. In: Mraovitch, S. and Sercombe, R. Eds., Neurophysiological Basis of Cerebral Blood Flow Control: An Introduction, John Libbley \& Co., London, 1334-1360.

[4] Olney, J.W., Ho, O.L. and Rhee, V. (1971) Cytotoxic effects of acidic and sulphur containing amino acids on the infant mouse central nervous system. Experimental Brain Research, 14, 61-76. doi:10.1007/BF00234911

[5] Lipton, S.A. and Rosenberg, R.A. (1994) Mechanisms of disease: Excitatory amino acids as a final common pathway in neurologic disorders. The New England Journal of Medicine, 330, 613-622. doi:10.1056/NEJM199403033300907

[6] Yu, T.X., Zhao, Y., Shi, W.C., Ma, R.D. and Yu, L.J. (1997) Effects of maternal oral administration of monosodium glutamate at a late stage of pregnancy on devel- 
oping mouse fetal brain. Brain Research, 747, 195-206. doi:10.1016/S0006-8993(96)01181-X

[7] Yu, L.J., Zhang, Y.P., Ma, R.D., Bao, L., Fang, J.Z. and $\mathrm{Yu}$, T.X. (2006) Potent protection of ferulic acid against excitotoxic effects of maternal intragastric administration of monosodium glutamate at a late stage of pregnancy on developing mouse fetal brain. European Neuropsychopharmacology, 16, 170-177. doi:10.1016/j.euroneuro.2005.08.006

[8] Ji, Y.B. (1999) Pharmacological action and application of blood-activating and stasis-eliminating available composition of traditional Chinese medicine. Heilongjiang Science and Technique Press, Harbin.

[9] Jing, H., Yao, L.Y., Li, J.S., Song, Y.Q. and Chao, W. (2002) Research progress of pharmacology of sodium ferulate. Northwest Pharmaceutical Journal, 17, 236-238.

[10] Grafe, E. (1992) Antioxidant potential of ferulic acid. Free Radical Biology and Medicine, 13, 435-448. doi:10.1016/0891-5849(92)90184-I

[11] Scott, B.C., Butler, J., Halliwell, B. and Aruoma, O.I. (1993) Evaluation of the antioxidant actions of ferulic acid and catechins. Free Radical Research Communications, 19, 241-253. doi:10.3109/10715769309056512

[12] Fernandez, M.A., Saenz, M.T. and Garcia, M.D. (1998) Anti-inflammatory activity in rats and mice of phenolic acids isolated from Scrophularia frutescens. Journal of Pharmacy and Pharmacology, 50, 1183-1186. doi:10.1111/j.2042-7158.1998.tb03332.x

[13] Ozaki, Y. (1992) Antiinflammatory effect of tetramethylpyrazine and ferulic acid. Chemical \& Pharmaceutical Bulletin, 40, 954-956. doi:10.1248/cpb.40.954

[14] Suzuki, A., Kagawa, D., Ochiai, R., Tokimitsu, I. and Saito, I. (2002) Green coffee bean extract and its metabolites have a hypotensive effect in spontaneously hypertensive rats. Hypertension Research, 25, 99-107. doi:10.1291/hypres.25.99

[15] Suzuki, A., Kagawa, D., Fujii, A., Ochiai, R., Tokimitsu, I. and Saito, I. (2002) Short- and long-term effects of ferulic acid on blood pressure in spontaneously hypertensive rats. American Journal of Hypertension, 15, 351-357. doi:10.1016/S0895-7061(01)02337-8

[16] Zhang, J., Jin, Q.Z. and Wang, X.G. (2007) Research progresses on synthesis and pharmacological activities of ferulic acid and its derivatives. Grain and Oil, 13, 43-45.

[17] Yan, J.J., Cho, J.Y., Kim, H.S., Kim, K.L., Jung, J.S., Huh, S.O., et al. (2001) Protection against beta-amyloid peptide toxicity in vivo with long-term administration of ferulic acid. British Journal of Pharmacology and Toxicology, 133, 89-96. doi:10.1038/sj.bjp.0704047

[18] Zhang, Y.P., Ma, R.D. and Yu, L.J. (2008) Sedative and hypnotic effect of sodium ferulate in mice. Nei Mongol
Journal of Traditional Chinese Medicine, 27, 9-11.

[19] Li, S.Z. (2004) Compendium of materia medica. People's Health Press, Beijing.

[20] Jiangsu College of New Medicine (1986) Dictionary of traditional Chinese and herbal medicine. Shanghai Science and Technology Publishing House, Shanghai.

[21] Liao, M.N., Wang, Y.P., Zhang, Y.P., Ma, R.D., Zhang, X.Y., Yu, L.J., et al. (2010) Neuroprotective and neurogenesis-enhancing effects of sodium ferulate. Chinese Journal of Cell Biology, 32, 840-849.

[22] Walsh, R.N. and Cummins, R.A. (1976) The open field test: A critical review. Psychological Bulletin, 83, 482504. doi:10.1037/0033-2909.83.3.482

[23] Xu, B.H., Duan, H.F. and Liu, R.Y. (1979) The effect on learing and memory of application of RNAase to hippocampus of rats. Chinese Science Bulletin, 24, 182-185.

[24] McDonald, J.W., Silverstein, F.S., Cardona, D., Hudson, C., Chen, R. and Johnston, M.V. (1990) Systemic administration of MK-801 protects against N-methyl-D-aspartate- and quisqualate-mediated neurotoxicity in perinatal rats. Neuroscience, 36, 589-599. doi:10.1016/0306-4522(90)90002-L

[25] McDonald, J.W., Silverstein, F.S. and Johnston, M.V. (1988) Comparison of neuroprotective effects of competitive and noncompetitive NMDA antagonists against NMDA mediated neurotoxicity in an in vivo perinatal rat model. In: Cavalheiro, E.A., Lehmann, J. and Turski, L. Eds., Recent Advances in Excitatory Amino Acid Research. Neurology and Neurobiology, Alan R. Liss, Inc. New York, 601-604.

[26] McDonald, J.W., Silverstein, F.S. and Johnston, M.V. (1989) Neuroprotective effects of MK-801, TCP, PCP and CPP against N-methyl-D-aspartate induced neurotoxicity in an in vivo perinatal rat model. Brain Research, 90, 33-40. doi:10.1016/0006-8993(89)90427-7

[27] McDonald, J.W., Uckele, J., Silverstein, F.S. and Johnston, M.V. (1989) HA-966 (1-hydroxy-3-aminopyrrolidone-2) selectively reduces N-methyl-D-aspartate (NMDA)-mediated brain damages. Neuroscience Letters, 104, 167-170. doi:10.1016/0304-3940(89)90349-2

[28] Tandon, P., Liu, Z., Stafstrom, C.E., Sarkisian, M., Werner, S.J., Mikati, M., et al. (1996) Long-term effects of excitatory amino acid antagonists NBQX and MK-801 on the developing brain. Developmental Brain Research, 95, 256-262. doi:10.1016/0165-3806(96)00094-6

[29] McDonald, J.W. and Johnston, M.V. (1990) Physiological and pathophysiological roles of excitatory amino acids during central nervous system development. Brain Research Reviews, 15, 41-70. doi:10.1016/0165-0173(90)90011-C 